\title{
Sur la production de l'hélium par le radium ${ }^{1}$
}

\author{
Par B. B. BOLTWOOD et E. RUTHERFORD
}

[Laboratoire de Physique de l'Université de Manchester.]

Introduction. - Le rapport qui existe entre l'hélium et les transformations des substances radioactives a constitué, durant les huit dernières années, un problème de grand intérêt et de haute importance, et a donné lieu à toute une série de recherches.

Rutherford et Soddy ${ }^{2}$ publièrent en 1902 leur théorie de désintégration de la matière radioactive et émirent l'hypothèse que l'hélium, qui se trouve en assez grande quantité dans les minéraux radioac. tifs, était un des produits de transformation des éléments radioactifs contenus dans ces minéraux. En 1903, Ramsay et Soddy ${ }^{3}$ montrèrent par l'expérience que l'hélium pouvait toujours être décelé en présence des sels de radium, et qu'il était aussi produit par l'émanation du radium; dans ces expériences, on n'a eu alfaire qu'à des quantités minimes d'hélium, et sa présence était démontrée au moyen de l'analyse spectrale. Peu après, ces observations furent confirmées par d'autres auteurs; M. Debierne trouva un peu plus tard que les préparations d'actinium produisaient aussi de l'hélium.

A cette époque, il était devenu très important de préciser la position de l'hélium dans la série générale des transformations radioactives. Rutherford avait montré en $1902^{4}$ que les rayons $\%$ du radium étaient des particules chargées positivement et émises avec une grande vitesse; la mesure du rapport $e / m$ de la charge d'une particule à sa masse conduisit à conclure qu'une particule $\alpha$ avait les dimensions d'un atome ordinaire, sa masse étant à peu près double de celle d'un atome d'hydrogène. I)ès le début, il a paru vraisemblable que si ces particules étaient constituées par une des substances connueś, celle-ci devait être soit de l'hydrogène, soit encore de l'hélium; et aussitôt que l'on eut découvert la production de l'hélium par le radium, Rutherford ${ }^{3}$ émit

1. Communication faite à l'Académie Impériale des Sciences de Vienne, le 16 mars 1911.

2. Phil. Mag., 4 (1902) 582.

5. Proc. Roy. Soc., 72 (1905) 204.

4. Phil. Mag., 5 (1903) 177.

5. Nature, 68 (1903) 566. l'opinion que la suarce de cette production devait être cherchée dans les particules $\%$; cette hypothèse, combinée avec les données numériques incomplètes qu'on possédait à ce moment, permit d'estimer que la quantité d'hélium produite en une année par un gramme de radium en équilibre devait être comprise entre 20 et $200 \mathrm{~mm}^{3}$.

L'importance qüil y avait à connaitre la vraie nature des particules $\alpha$ fit procéder à des mesures plus précises de leur vitesse et du rapport $e / m$; ce dernier se trouva, d'après Rutherford ${ }^{1}$, être égal à $5,07.10^{5}$ U. E. M. Ceci indiquait que les particules étaient soit des molécules d'hydrogène portant une seule charge élémentaire, soit des atomes d'hélium avec une charge double; la seconde supposition semblait la plus probable. L'étude de la question fut poussée plus loin par Rutherford et Geiger ${ }^{2}$, lorsqu'ils eurent trouvé une méthode pour la numération directe des particules $\%$; leurs expériences amenèrent à la conclusion que la charge d'une particule est bien égale au double de la charge élémentaire, soit à $9,3 \cdot 10^{-10}$ U. E. S. Finalement, la preuve décisive do l'ideutité d'une particule $\alpha$ arec un atome d'hélium fut donnée par Rutherford et Royds ${ }^{5}$, qui montrèrent que lorsque des particules $\alpha$ pénètrent dans un vide ou dans une paroi solide, il s'y accumule toujours de l'hélium. Il a été ainsi démontré péremptoirement que la particule $\alpha$ est un atome d'hélium projeté, portant avec lui deux charges élémentaires.

Rutherford et Geiger ${ }^{4}$ purent alors calculer, d'une facon plus exacte, la production d'hélium à laquelle on devait s'attendre théoriquement. Un gramme de radium en équilibre avec ses produits émet par seconde $13,6.10^{10}$ particules $\alpha$, c'est-à-dire autant d'atomes d'hélium. Si l'on admet que la charge d'un atome d'hydrogène est de $1,63.10^{-10}$ U. E. S., on calcule aussitôt, à p.rrlir de léquivalent électrochimique de l'hydrogène, qu'un centimètre cube de

1. Plit. Mag., $12(1906) 5 \% 8$.

2. Proc. Roy. Soc., 81 (1908) 141.

5. Phil. Mag., 17 (1909) 281.

4. Proc. Roy. Soc., 81 (1908) 162. 
n’importe quel gaz contient 2,72.1019 molécules à la ld pression et à la lempérature normales; l'hélium élant monoatomique, la quantité de ce gaz produite par un gramme de radium en équilibre doit donc être égale à $\frac{15,6 \cdot 10^{10}}{2,7 \grave{2} \cdot 10^{19}} \mathrm{~cm}^{\overline{5}}$ par seconde, soit 158 millimètres cubes par an.

La première mesure directe de celle production a été faite par sir James Dewar ${ }^{1}$, sur $70 \mathrm{mgr}$ de chlorure de radium anhydre; cet échantillon constituait une partie de la substance préparée par $\mathrm{M}$. Thorpe lors de sa délermination du poids atomique du radium. Le sel cristallisé était enfermé dans un vase évacué et réuni à une jauge de Mac Leod; on chauffait le sel de temps en temps pour en extraire l'hélium accumulé. Les autres gaz pouvant être présents élaient éliminés cn les absorbant par un morceau de charbon de noix de coco refroidi dans de l'air liquide. La quantité d'hydrogène présente dans le mélange put ìtre estimée en refroidissant le charbon jusquà la température de l'hydrogène liquide.

Dans sa première communication, M. Dewar avait conclu qu'un gramme de Ra en équilibre produisait par jour $0,57 \mathrm{~mm}^{\overline{3}}$ d'hélium; dans une expérience ultérieure², ò̀ le sel était resté enfermé pendant 9 mois, la production mojenne a été de $0,163 \mathrm{~mm}^{3}$ par jour. Le nombre $0,57 \mathrm{~mm}^{5}$ donné dans la première communication s'est trouré être fautif, par suite d'une erreur de calcul; le nombre corrigé est 0, $199 \mathrm{~mm}^{3}$ par jour. La produclion rapportée à un gramme et à un an a donc été de $182 \mathrm{~mm}^{5}$ dans la première expérience, et $169 \mathrm{~mm}^{5}$ dans la scconde; les deux nombres sont un peu plus élevés que la valeur théorique, soit $158 \mathrm{~mm}^{3}$.

Comme il est très important de connaître la valeur de cette production avec toute la précision possible, les auteurs en ont entrepris une nouvelle détermination; ils ont fait deux séries de mesures indépendantes, en se scrvant d'une partie considérable de l'échantillon prêté obligeamment à l'un d'eux par l'Académic de Vienne.

Purification de l'échantillon. - Il importait que le sel de radium employé ne contìnt aucune substance radioactive autre que le radium lui-même; ce sel itait un chlorure de baryum-radium, à 7 pour 100 environ de radium; il arait déjà subi une cristallisation fractionnce partielle avant d'arriver dans nos mains. Il était donc très improbable qu'il pût contenir d'autres substances radioactives que le radium et ses produits. Avant les expériences, le sel avait été conservé pendant plus d'un an en solution aqueuse légèrement acide; il avait existé précédemment sous forme de cristaux, durant un

1. Proc. Roy. Soc., $81(1908) 280$.

2. Proc. Roy. Soc., 83 (1910) 410. intervalle de temps mal défini. I'our ćliminer le radioplomb et le polonium qui s'étaicul accumulés pendant ce temps, on a opéré de la façon suivante. Un a dissous $50 \mathrm{mgr}$ de trichlorure d'antimoine, $10 \mathrm{mgr}$ de nitrate de plomb et $5 \mathrm{mgr}$ de nitrate de bismuth dans un peu d'acide chlorhydrique diluć, et on a ajoulé cette solution à celle du chlorure radifìre: le tout a été étendu d'eau jusqu'à un volume de $100 \mathrm{~cm}^{5}$, et un excès d'hydrogìne sulfuré a été introduit. Le précipité des sulfures se trouva coagulé cn préscnce du sel d'antimoine et put ètrc facilement séparé par filtration; il a été lavé soigneusement avec de l'eau distillće, puis dissous à chaud sur le filtre par de l'acide chlorhydrique dilué, auquel on avait ajouté quelques cristaux de chlorate de potasse. $\Lambda$ près avoir étendu avec de l'eau, on répéta le traitement far l'hydrogène sulfuré; la liqueur recueillic après la filtration fut ajoutće à celle de la première filtration. L'adjonction des sels de plomb et de bismuth à la solution primitive avait eu pour bat d'assurer la précipitation complète du radioplomb et du prolonium ${ }^{1}$.

L'ensemble des deux solutions filtrées après précipitation par $\mathrm{H}^{2} \mathrm{~S}$ fut évaporé à sec dans une capsule de quartz, et le résidu chauffé à feu lent, pour éliminer l'excès d'eau ef d'acide chlorhydrique. Le scl sec fut introduit dans une capsule cylindrique en platine, munie d'un couvercle percé d'un trou. Cette capsule fut enfermée dans un tube de verre d'Iéna (fig. 1)

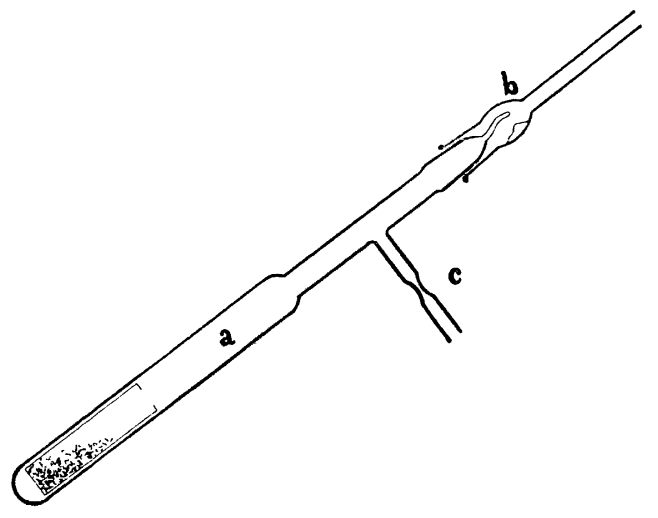

Fig. 1.

dont l'une des extrémités, étirée en capillairc mince, pouvait être introduite, au moyen d'un rodage, dans le bout $b$ d'un tube de verre ordinaire. Cne saillie intéricure dans le tube $b$ permettail au besoin do briser le bout du tube $a$ en tournant celui-ci dans le rodage, et d'établir ainsi une communication entre $a$ et $b$, pour retirer l'hélium de $a$ à la fin de la période d'accumulation. Le tube a était vidí complètement à l'aide du tube litéral $c$, fermé ensuite à la flamme.

1. Bulıwood, Amer. Journ. of Science, 25 (1908) 228. 
Dosage du radium dans le sel. - La quanlité de radium contenue dans le sel a été déterminée par une mesure de son rayonnement $\gamma$, plus de deux mois après la fermeture du tube; ce rayonnement a été comparé, à l'aide d'un électroscope à parois de plomb épaissss, avec le rayonnement $\gamma$ d'un étalon de notre laboratoire contenant $5,69 \mathrm{mgr}$ de $\mathrm{RaBr}^{2}$; l'opération a été faite d'une part dircetement, d'autre part par comparaison avec un troisième échantillon dont le contenu a été déterminé à l'aide du petit ćtalon et correspondait à $52 \mathrm{mgr}$ de $\mathrm{RaBr}^{2}$. Les mesures ont été faites en disposant les échantillons à différentes distances et dans différentes conditions expérimentales; le résultat en a étc que le tube employé dans ces expériences contenait $191 \mathrm{mgr}$ de Ra (élément), ce qui correspond à $526 \mathrm{mgr}$ de RaBr².

Lorsque les expériences sur la production d'hélium furent achevées, le radium présent dans le sel a été dosé encore une fois de la manière suivante. Le scl a été dissous dans de l'eau distillée additionnée d'un peu d'acide chlorhydrique, et la solution étendue à un volume de $50 \mathrm{~cm}^{3}$. Une faible portion bien définie de la solution $(1,22$ pour 100$)$ a été prélevée, évaporée à sec dans un petit tube de rerre et enfermée hermétiquement à la flamme. Dans un mois, cette portion a été comparće directement, par la méthode des rayons $\gamma$, avec l'étalon contenant $5,69 \mathrm{mgr}$ de $\mathrm{RaBr}^{2}$, et s'est trouvée être équivalente à $4,02 \mathrm{mgr}$ de $\mathrm{RaBr}^{2}$. D'après ceci, la quantité totale de Ra présente primitivement dans le sel employé aurait été égale à $193 \mathrm{mgr}$.

Accumulation et mesure de l'hẻlium. La quantité d'hélium produite par le sel de radium a étć accumulée, purifiće et mesurée dans l'apparcil représenté schématiquement sur la figure 2. Cet appareil pouvait être évacué à l'aide d'une pompe à mercure $\mathbf{P}$ qui en faisait parlic; les gaz évacués pouvaient être recucillis en D. Le tube $\mathrm{M}$ contenait du $\mathrm{P}^{2} 0^{3}$ comme desséchant; lo tube $\mathrm{A}$ avec ses appendices capillaires $\mathrm{E}$ et $e$ était essentiellement une jauge MacLcod, mais il é:ait employé à. la mesure de petits volumes, au licu de l'être, comme d'habitude, à celle des pressions.

Le volume du tube capillaire $\mathbf{E}$ était exactement déterminé en chaque partie de sa longueur, en le calibrant avec un fil de mercure; lc tulee $e$ était fait du même morceau de verre que $\mathrm{E}$ et avait par conséquent la mème section, de sorte qu'il n'intervenait aucune correction de capillarité dans la lecture de la pression en E.

Deux tubes débouchient latéralement dans 1 et pouvaient être fermés par des soupapes à flotteurs de verre; le flotteur du tube inférieur était du modèle ordinaire, celui d'en haut avait une construction spéciale qui empèchail que du gaz pùt être pris entre le llotleur et la paroi extérieure. Le lube inféricur se prolongeait jusqu'au tube $M$ à $\mathrm{P}^{2} \mathrm{O}^{\prime \prime}$; il était muni d'un robinet en $a$ et réuni à un tube $\mathrm{C}$ contenant plusieurs grammes de charbon de noix de coco. Lin tube latéral

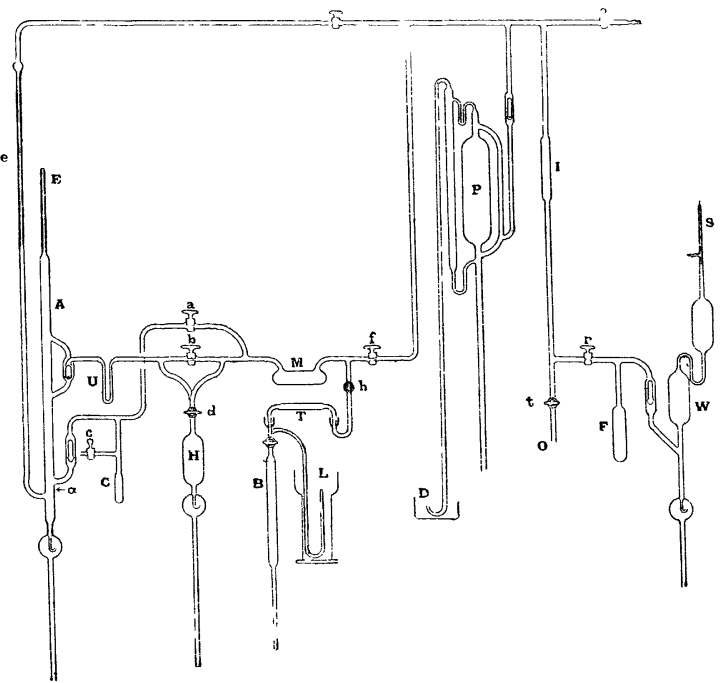

Fig. 2.

$c$ permettait d'introduire en cet endroit de petits volumes d'oxygène électrolytique, pur el sec. Le tube supéricur aboutissant en $I$ avait un prolongement en forme d'U (marqué $\mathrm{C}$ sur la figure) en verre mince; ce tube se continuait ensuite par le robinet $b$ jusqu'ì la pompe auxiliaire $\mathrm{H}$, laquelle servait à extraire le gaz de $\mathrm{M}$ et à le refouler dans $\mathrm{A}$ et dans ses communications.

La partie de l'appareil adjacente était une burette B munie d'électrodes de platine destinées à faire passer des étincelles; cette burette servait à introduire les gaz à l'aide du tube $L$ se terminant sous mercure. Après avoir été soumis à l'action de l’ćtincelle, les gaz étaient envojés à travers un tube $\mathrm{T}$ de verre dur contenant de l'oxyde de cuivre chauffé au rouge.

Le reste de l'appareil, représenté par la partie droite de la figure, servait à examiner le spectre du gaz; il permettait de retirer de la partie A tout le gaz dont on venait de mesurer le volume et de l'introduire dans le petit tube à électrodes $S$, où son spectre pouvait être étudié à loisir.

Les rícipients A, II, B, etc. étaient munis de purgeurs Bunsen destinés à empècher l'air de pénétrer le long des colonnes de mercure. Les tubes de verre réunis à ces purgeurs se prolongeaient vers le bas sur une longueur supérieure à $76 \mathrm{~cm}$; les extrémités inférieures, ainsi que les réservoirs de mercure correspondants, ne sont pas représentés sur la ligure.

Étalonnement $d u$ tube servant à mesurer le volume du gaz. - Pour calibrer la jauge $\Lambda$ destinée aux mesures des volumes, on commençail par vider tout l'apparcil à l'aide de la pompe $\mathrm{P}$, jus- 
qu'à la limite extrême de la raréfaction, tout en chauffant le tube à charbon $\mathrm{C}$, afin d'en expulser les gaz absorbés. On faisait alors monter lc mercure en A jusqu'au point $\alpha$, en fermant les robinets $f, d, a, c$, et on introduisait par $\mathrm{L}$ une petite quantité d'oxygène mélangée avec un peu d'hélium pur (obtenu à partir de l'uraninite cristalline). On fermait alors $b$ et on entourait $\mathrm{C}$ d'air liquide; en 20 minutes environ, on faisait monter le mercure en $\mathbf{A}$ et on mesurait le volume et la pression du gaz dans le tube capillaire $\mathbf{E}$. Après avoir élevé le mercure encore une fois jusqu'au bord supérieur de $\mathrm{A}$, on ouvrait les robinets $a, b, f$, on enlevait l'air liquide, on attendait que le tube $\mathrm{C}$ cût pris la température de la pièce; on le chauflait ensuite au rouge sombre et on faisait le vide complet dans $\mathrm{C}, \mathrm{U}, \mathrm{M}$, etc. Les robinets $a, b$ étaient alors refermés, les tubes $C$ et $U$ entourés d'air liquide, et le mercure dans A baissé jusqu'en $\alpha$; l'hélium, cmprisonné auparavant en $\mathrm{E}$, se distribuait maintenant dans E, A, C, U; on élevait de nouveau le mercure en A et on mesurait le volume de l'hélium enfermé cette fois dans E. Le rapport de ce volume à celui mesuré précédemment est évidemment égal au rapport de la quantité d'hélium emprisonné dans le tube E lorsqu'on élève le mercure en $A$, à la quantité totale contenue dans A et dans ses communications; un grand nombre d'expériences concordantes ont montré que ce rapport était égal à 71,5 pour 100 .

En faisant en A le vide extrême que pouvait donner la pompe $\mathrm{P}$, en fermant les robinets $a, b, c$ et en plongeant $G$ dans l'air liquide pendant au moins vingt minutes, il était possible d'obtenir en A une pression tellement basse qu'il ne se faisait en $\mathrm{E}$ aucune augmentation de pression appréciable, lorsqu'on y faisait monter le mercure jusqu'à $1 \mathrm{~mm}$ de l'extrémité supérieure; dans ces conditions, il a été possible d'établir la correction nécessitéc par les petites différences dans les diamètres des tubes $\mathbf{E}$ et $e$; il a été trouvé de la sorte que lorsque le tube $\mathbf{E}$ contenait du gaz, l'erreur avec laquelle sa pression était déterminée par la mesure à la jauge, était inférieure à un demi-millimètre de mercure.

Quantité d'hélium dégagée par le radium. Première détermination. - Le sel de Ra-Ba décrit plus haut fut maintenu pendant quatre-vingttrois jours dans le tube représenté sur la figure 1; ce sel contenait encore une partis de son eau de cristallisation. A l'endroit 0 de l'appareil principal (fig. 2), on souda d'abord un petit ballon avec de l'anhydride phosphorique, puis un autre avec de la potasse solide; on y adjoignit alors le tube $b$ de la figure 1 , et on fit le vide sur le tout, jusqu'au tube capillaire qui fermait toujours encore la communication entre $a$ et $b$; les robinets $r$ et $/$ étaient fermés pendant ce temps. Le tube capillaire fut alors cassé à l'intérieur du rodage, et les gaz contenus dans a furent enlevés par la trompe et recueillis en D. Durant cette opération, la partic inféricure de $a$, avec la capsule de platine qu'elle contenait, étaient chauffées au rouge, pendant une demi-heure, a l'aide d'un chalumeau, pour obtenir un dégagement complet des gaz occlus: l'eau de cristallisation était dégagée en même temps et allait s'absorber dans les tubes à potasse et à $\mathrm{P}^{2} 0^{3}$. Une petite quantité d'oxygène électrolylique fut ajoutée au mélange gazeux, et celui-ci fut introduit en $\mathrm{B}$ à travers $\mathrm{L}$; on fit agir l'étincelle, et on refoula en A le gaz restant, en lui faisant traverser lentement le tube $\mathbf{U}$, plongé dans l'air liquide. L émanation contenue dans le mélange était, bien entendu, condensée dans le tube $\mathrm{U}$.

Lorsque le gaz eut été transporté en A, on entoura d'air liquide le tube à c'ıarbon $\mathrm{C}$, le niveau du mercure étant en $\alpha$; dans vingt minutes, on fit monter le mercure en $\mathrm{E}$ et on mesura le volume et la pression du gaz ainsi emprisonné. Le niveau du mercure fut alors amené au-dessous de l'endroit où le tube $\mathrm{U}$ débouchait dans A; les gaz non condensés contenus dans $\mathrm{C}$ et dans les tubes de communication furent recueillis par la trompe auxiliaire $H$ et renvoyés ensuite dans A. Après avoir fermé les robinets $b$ et $d$ et ouvert $a$, on enleva l'air liquide qui refroidissait le tube $\mathrm{G}$, on chauffa ce dernier et on enleva, à l'aide de la pompe principale, les gaz qui avaient été condensés en $\mathrm{C}$.

Le robinet $a$ fut alors fermé, le tube C plongé de nouveau dans l'air liquide et le niveau du mercure dans A ramené en $x$; les gaz contenus en A étaient donc de nouveau exposés à l'action du charbon refroidi. Dans trente minutes environ, on fit remonter le mercure et on mesura le volume et la pression du gaz contenu en $\mathrm{E}$; les gaz non condensés contenus en G furent de nouveau transportés en A, l'air liquide enlevé et le tube $\mathrm{C}$ chauffé; les gaz condensables furent de nouveau enlevés à la trompe. Ce cycle d'opérations fut répété cinq fois de suite et fournit ainsi cinq mesures distinctes du volume et de la pression. La deuxième et la troisième firent voir une faible diminution de la quantité du gaz; mais les trois dernières ont été d'accord entre elles à 1 pour 100 près; le purification de l'hélium avait donc été poussée aussi loin que cela est possible à l'aide du charbon refroidi à basse température.

L'hélium recueilli dans l'appareil fut ensuite trans. porté dans le tube à électrodes $\mathrm{S}$; le spectre obtenı était essentiellement un spectre pur d'hélium.

I)ans les expériences qui viennent d'être décrites, les seules précautions prises pour èliminer l'hydrogène étaient l'action de l'étincelle en présence d'oxygène pur, et l'exposition lu gaz restant à l'action du charbon refroidi. Le tube à oxyde de cuivre fut ajouté plus tard, en considération de ce fait (établi par 
M. Dewar el par d'aulres auleurs), que le traitemenl au charbon refroidi ne sulfit pas, dans de's conditions analogue, ¿̀ éloigner les derniòres traces d'hydrogìne.

Une expérience spéciale a été faite pour voir à quel degré la présence d'hydrogène pouvait vicier les résultats des mesures. A cet effet, on introduisit dans l'appareil une pelite quantité d'hydrogène, et on lui fit subir plusieurs fois de suite le même cycle d'opéralions qu'aux gaz dégagés par le sel radioactif; on trouva que la quantité restée après le premier traitement par le charbon refroidi diminua rapidement au cours des opérations suivantes, et fut réduite, après trois cycles complets, à moins de 1 pour 100 de la valeur trouvée précédemment pour la quantité d'hélium dégagée par le radium. Il n’était donc pas vraisemblable que la présence d'hydrogène eût pu introduire une erreur sérieuse.

Il y avait à se demander encore si la chauffe dı sel radifìre avait suffi pour en extraire complètement tout l'hélium formé et, d'autre part, si le sel solide n’avait pas dégagé b aucoup d'émanation pendant le temps où il élait resté enfermé; car s’il en avait dégagé, cetle émanation se scrait distribuée dans tout le tube, ct les particules $\alpha$ produites par sa désagrégration auraient en grande partie pénétré dans les parois et causeraient ainsi une erreur difficile à éviter. Pour répondre à ces questions, on mesura le rayonnement $\gamma$ de la partie supérieure du tube $a$ (fig. 1) arant que celui-ci fùt chaulfé; le rayonnement de la partie inférieure était, pendant cette mesure, arrêté par de gros blocs de plomb. La mesure montra que la quantité d'émanation dégagée à froid était entièrcment négligeable; car aucun rayonnement ne fut observé, quoique l’appareil eût pu déceler une quantité d'émanation égale à 1 pour 100 de la quantité tola'c. Une autre série de mesure's de l'activité $\gamma$ a été eflectuée aussitôt après la chauffe du sel; on s'assura ainsi que toute l'émanation avait été extraite par la chauffe; il était permis d'admettre, par conséquent, que l’hélium était également dégagé en totalité.

Quantité d'hélium dégagée par le sel de radium. Deuxième détermination. -- Afin de ne point laisser subsister de doutes sur l'influence de l'hydrogìne libre, et aussi pour ériter la possibilité d'une extraction d'hélium incomplète, la seconde détermination a été faite dans des conditions entièrement différentes. Le tube $\mathbf{T}$ à oxyde de cuivre fut adjoint à l'appareil, et l'on s'assura, par une expérience préalable, que le passage d'un mélange. gazeux ì travers la couche de CuO, chauffée à un roage modéré, sutfisait pour absorber les dernic̀res traces d'hydrogène. Le sel de radium fut enfermé cette lois pendant 152 jours, à l'expiration desquels les gaz présents en $a$ furent enlevés et recueillis à l'aide de la

T. 8. trompe; ces gaz consistaient principalement cn oxygìne, dont on avait rempli le tuhe $a$ avant de le fermer. La capsule contenant le sel fut alors retirée du tube $a$ et introduite dans un autre tube semhable (fig. 5), après qu'on eut enlevé le couvercle

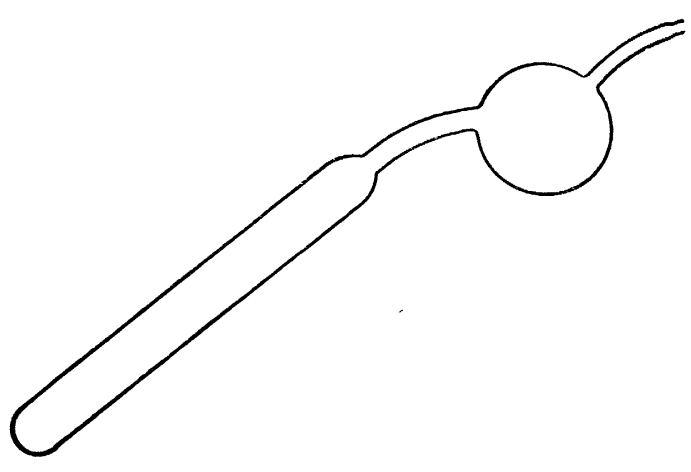

Fig. 3.

percé dont la capsule était précédemment recouverte. La capsule était posée sur le fond de la partie cylindrique du tube: la partie inférieure de celui-ci fut enveloppée de papier mouillé, pour la protéger contre un échauffement éventucl, et la partie supérieure du tube, munie d'un renflement sphérique, fut firmée; ce reaflement servait à empêcher que des gouttelettes de la solution radifère que la capsule devait contenir plus tard, pussent être projetées au dehors pendant l'ébullition qui allait se produire sous pression réduite.

Le tube fut fermé par un bout de caoutchouc à vide serré par une pince à vis ; après évacuation complète du tube, on introduisit, à travers le caoutchouc, $50 \mathrm{~cm}^{3}$ environ d'acide chlorhydrique dilué, de telle façon qu'aucune trace d'air ne pouvait entrer en même temps. Le sel fut alors dissous entièrement en chauffant légèrement le tube, et les gaz furent extraits à la pompe, à travers de petils tules à potasse et à $\mathrm{P}^{2} 0^{*}$, et ensuite un travers un petit tube $\mathrm{U}$ refroidi par l'air liquide, ceci afin de retenir l'émanation au passage. Le gaz ainsi recueilli fut réuni à celui qu'on avait retiré en chauffant le sel, et le tout fut introduit dans la burette $B$. Après avoir subi l'action de l'étincelle, le gaz fut introduit dans l'appareil de mesure à travers le tube à $\mathrm{Cu} 0$ chauffé; la mesure de la quantité d'hélium fut effectuée de la même facon qu'auparavant. On exposa le gaz trois fois de suite à l'action du charbon refroidi, en enlevant, après chaque mesure, les gaz qui pouvaient être condensés; les résultats furent parfaitement concordants, et l'on n'observa pas la diminution de volume qui s'était produite dans la première série d'expériences, où l'hydrogène n'avait pas été complèment éliminé lors de la première mesure. Les tableaux suivants contiennent les résultats des deux séries. 
Première détermination (temps d'accumulation : $8 \bar{j}$ jours).

$\begin{array}{cc}\text { Numéro de la mesure. } & \text { Volume d'lélium à } 0^{\circ} \mathrm{el} 760 \mathrm{~mm} . \\ 1 & 8,08 \mathrm{~mm}^{\top} \\ 2 & 6,79 \\ 3 & 6,59 \\ 4 & 6,54 \\ 5 & 6,60 \\ \text { Moyenne des mesures } 5,4,5: & 6,58\end{array}$

Deuxième détermination (temps d'accumulation : 152 jours).

Numéro de la mecure.

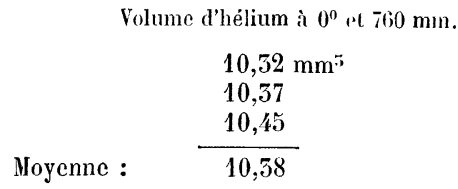

Calcul de la production d'hélium. - Soit $x$ le volume d'hélium produit par jour par le radium (élément) contenu dans le sel, et $y$ le volume d'hélium produit par jour par la quantité d'émanation et de ses deux produits à rayons $\alpha$ ( $\mathrm{RaA}$ et $\mathrm{RaC}$ ) qui serait en équilibre avec ce radium; si les trois produits (émanation, RaA, RaC) sont en équilibre entre cux, on a

$$
y=5 x,
$$

c'est-à-dire que les trois produits donnent naissance à trois fois autant d'hélium que le radium seul. Toutefois, dans nos expériences, le sel était privé d'ćmanation au début de l'accumulation; la quantité totale d'He produite pendant une accumulation de $\tau$ jours devail done être :

$$
\begin{aligned}
Q= & \tau x+y \int_{0}^{\tau}\left(1-e^{-\lambda t}\right) d l=\tau x+\left(\tau-\frac{e^{-\lambda \tau}}{\lambda}\right) ! y \\
& =\tau x+\left(\tau-\frac{1}{\lambda}\right) y=\left[\tau+5\left(\tau-\frac{1}{\lambda}\right)\right] x .
\end{aligned}
$$

$\lambda$ est ici la constante radioactive de l'émanation, et le jour est pris comme unité de temps; on a posé $e^{-\lambda \tau}=1$, ce qui est exact avec une grande approximation pour $\tau>40$ jours.

Les valeurs de $Q$ et de $\tau$ étant connues pour cha cune des expériences, on trouve

$$
\begin{aligned}
& x=0,0209 \mathrm{~mm}^{3} \text { dans la première série, } \\
& x=0,0205 \mathrm{~mm}^{3} \text { dans la seconde série, }
\end{aligned}
$$

soit en moyenne $0,0206 \mathrm{~mm}^{\overline{5}}$ par jour. La quantité de Ra dans le sel ayant été égale à $192 \mathrm{mgr}$ (moyenne de 191 et 193, v. plus haut), la production par jour et par gramme de Ra privé de ses produits est done

$$
\frac{0,0206}{0,192}=0,107 \mathrm{~mm}^{3} \text {, }
$$

et la productiou par an el par ogr. de Ra en équilibre avec ses produits ì évolulion rapide (émanation, RaA, RaC) :

$0,107 \times 565 \times 4=156 \mathrm{~mm}^{3}$ dhélium par an.

Cette valeur, donnée par l'expérience, concorde très bien avec la valeur calculéc $\left(158 \mathrm{~mm}^{5}\right)$ dont il a été question plus haut; les considérations sur lesquelles le calcul avait été fondé sont donc confirmées d'unc façon complète.

Il n'y a actuellement plus de doute que la particule $\alpha$ ne soit un atome d'hélium avec une charge élímentaire double, et que l'hélium lui-même ne soit monoatomique. L'accord constaté entre le calcul et la théoric confirme d'une façon remarquable le bien fondé de la conception atomistique de la matière. Le nombre des particules $\alpha$ émises par seconde par un gramme de radium a été compté directement, et le volume correspondant d'hélium a été déterminé expérimentalement; ces deux expériences permettent de calculer, avec un minimum de suppositions, le nombre d'alomes d'hélium contenus dans $1 \mathrm{~cm}^{5}$ à $0^{n}$ et à la pression normale; ce " nombre de Loschmidt " se trouve être égal à $2,69.10^{19}$. Suivant l'hypothèse d'Avogadro, c'est aussi le nombre d'atomes de n'importe quel autre gaz, pris dans les conditions normales.

On voit que cette méthode n'implique pas la connaissance de la charge élémentaire $e$; au contraire, la valeur de $e$ peut en être déduite immédiatement.

Il importe de remarquer que l'accord entre la valeur théorique de la production d'hélium et la valeur observéz ne dépend pas de l'exactitude de l'étalon de radium employé, car c'est le même étalon qui a été utilisé dans les deux cas. Dès qu'on aura établi un étalon international de radioactivité, il sera facile de calculer la production d'hélium sur la base de. ce nouvel étalon.

Gràce à l'obligeance de M. J. Dewar, nous avons été à même de comparer l'échantillon de radium employé dans ses expériences à notre étalon provisoire; il s'est trouvé que la quantité du radium utiisé par M. Dewar et estimée par lui à $70 \mathrm{mgr}$ de IRa Cl² pur, étail équivalente à $72 \mathrm{mgr}$ de Ra $\mathrm{Cl}^{2}$ d'après notre étalon. En ramenant à notre étalon la production d'hélium observée par M. Dewar, elle se réduirait donc a $164 \mathrm{~mm}^{-3}$ par an au lieu de $169 \mathrm{~mm}^{5}$; cc nombre est un peu plus élevé que le nòtre, mais les deux s'accordent en principe.

Production d'hélium par l'émanation du radium. - Il a ćté également fait une mesure de la quantité d'hélium formée pendant la destruction d'une quantité connue d'émanation du radium. L'émanation, extraite d'une solution de radium, a été séparée et purifiée par condensation à la température de l'air liquide. On prépara un tube en verre fusible de 
$10 \mathrm{~mm}$ de diamètre intérieur el de $8 \mathrm{~cm}$ de long; un bout de ce tube portait un joint rodé muni d'un bout capillaire, comme dans le cas du tube a de la figure 1; ce joint s'adaptait à un autre tube, anilogue au tube $b$ de la mème figure.

Le tube de verre a été rempli par du soufre fondu; il a été évacué pendant que le soufre se refroidissait. Après avoir introduit l'émanation purifiée dans ce tube, on scella le dernicr, puis on le chauffa jusqu'à la fusion du soufre, tout en le tournant continuellement, de manière à obtenir sur les parois une couche uniforme de soufre, de $5 \mathrm{~mm}$ d'épaisseur environ. Cette couche de soufre devait former la paroi intérieure dans laquelle seraient venues se planter les particules \%. émises par l'émanation et le dépôt actif, et dont on aurait pu ensuite extraire facilement l'hélium en faisant fondre le soufre.

Environ 19 heures et demie après l'introduction de l'émanation, son rayonnement $\gamma$ a été mesuré et comparé à celui de l'étalon de radium; il en résulta que l'émanation présente dans le tube au début était équivalente à la quantité qui est en équilibre avec $126 \mathrm{mgr}$ de Ra.

Vingt-et-un jour après l'introduction de l'émanation, on chauffa le tube afin de faire fondre le soufre, jusqu'au moment où le bout capillaire devint complètement transparent. Le tube fut alors adapté à l'appareil de mesure au moyen du rodage, l'appareil évacué jusqu'au rodage, le bout capillaire cassé, et les gaz retirés et recueillis pendant qu'on chauffait le tube de manière à maintenir le soufre fondu. On laissa alors refroidir le tube et on introduisit un peu d'oxygène qui fut ensuite retiré à la pompe et ajouté aux autres gaz recueillis. Les gaz furent introduits dans la burette $\mathrm{B}$ (fig. 2), conduits à travers de l'oxyde de cuivre chauffé, puis refoulés dans l'appareil de mesure; le volume de l'hélium fut déterminé suivant la méthode habituelle. Le gaz fut alors transporté dans le tube $S$, où son spectre a été examiné et reconnu pour un spectre pur d'hélium. Le volume d'hélium trouvé a été égal à $0,202 \mathrm{~mm}^{5}$.

La quantité d'émanation présente au début dans le tube a été celle en équilibre avec $0,196 \mathrm{gr}$ de Ra; la production initiale d'hélium devait donc se faire à raison de

$$
0,126 \times 5 \times 0,107 \mathrm{~mm}^{\overline{5}} \text { par jour, }
$$

le facteur 5 étant ajouté pour tenir compte de l'existence de trois produits à rayons $\alpha$ : émanation, . RaA et Ral. Mais comme l'émanation se détruit avec le temps, la production d'hélium doit diminuer, et la quantité totale formée sera proportionnelle à l'expression

$$
\frac{1}{\lambda}\left(1-e^{-i l}\right),
$$

où $\lambda$ est la constante radioactive et $t$ le temps. Si nous adoptons pour $\lambda$ la valeur qui correspond à une période de 5,85 jours et que nous choisissions le jour pour unité de temps, l'expression ci-dessus prend, pour $l=21$, la valeur 5 , 42 ; c'est-à-dire que la quantité d'hélium produite en 21 jours sera

$$
0,126 \times 5 \times 0,107 \times 5,42=0,220 \mathrm{~mm}^{3} .
$$

Si l'on tient compte des difficultés de l'expérience, on voit que ce nombre s'accorde bien arec la valeur observée.

Production d'hélium par le polonium. - On a déterminé aussi la production d'hélium par le polonium. Les sulfures de plomb, de bismuth et d'antimoine, obtenus lors de la purification du sel de radium, furent décomposés en les digérant avec de l'acide chlorhydrique concentré et un peu de chlorate de potasse. La solution fut chauffée jusqu'à évaporation de la majeure partie de l'excès d'acide chlorhydrique dilué. Cette solution fut versée dans une solution concentrée de sulfure d'ammoniaque; les sulfures de plomb et de bismuth furent séparés et traités à nouveau par l'acide chlorhydrique et le chlorate de potassc. On ajouta de l'acide sulfurique en excès, on concentra le mélange par ẹvaporation et on chauffa jusqu'à apparition des vapeurs d' $\mathrm{H}^{2} \mathrm{~S} \mathrm{O}^{\prime}$. Après refroidissement, on ajouta au résidu un peu $\mathrm{d}^{1} \mathrm{H}^{2} \mathrm{SO}^{4}$ étendu et l'on sépara le sulfate de plomb insoluble, sur un petit filtre en amiante. Le sulfate de plomb fut alors traité à chaud par HCl concentré et par $\mathrm{KCl}^{5}$; de l'acide sulfurique dilué fut ajouté à la solution et les opérations qui viennent d'être décrites furent répétées encore une fois. On réunit alors les deux solutions obtenues par filtration de sulfate de plomb, on chauffa jusqu'à ébullition et on ajouta un petit excès d'ammoniaque. Le faible précipité de bismulh fut séparé par filtration et dissous dans HCl dilué. Le volume de cette solution fut mesuré exactement, et unc faible portion bien définie en fut prélevée. Dans la solution principale, on introduisit $5 \mathrm{gr}$. de cuivre métallique en petits morceaux; le mélange fut chauffé doucement et conservé pendant plusieurs heures en le remuant souvent. Le polonium présent dans la solution chlorhydrique se déposa pendant ce temps sur le cuivre, en couche bien adbérente; le cuivre fut alors lavé et séché.

Ce cuivre arce sa couche de polonium fut introduit dans un tube de verre, semblable au tube $a$ de lat figure 1, mais plus petit; ce tube fut évacué et de l'oxygène fut introduit; on scella ensuite le tube.

128 jours plus tard, ce tube à polonium fut relié à l'appareil de mesure et les gaz retirés à la pompe, pendant qu'on chauffait au rouge vif l'extrémité inférieure du tube, pour chasser l'hélium occlus dans le métal. L'hélium présent dans le mélange fut dosé suivant la méthode habituelle; on en trouva $0,009 \mathrm{~mm}^{5}$, 
Le gaz put facilement être identifié par son spectre.

La fraction de la solution polonifìre qui avait été prélerée avant le traitement par le cuivre, fut étendue à $10 \mathrm{~cm}^{3}$ par de l'eau lígèrement acidulée avec $\mathrm{HCl}$; cette solution fut introduile dans un vase de verre dont le fond consistait en une lame de cuivre. Le polonium se déposa sur cette lame, et le nombre des particules $\alpha$ qu'il émettait fut obligeamment déterminé pour nous par M. Geiger; ce nombre était de $10,7.10^{5}$ par seconde. La solution contenait $1 / 151 \mathrm{de}$ la solution principale: celle-ci aurait done émis $14,0.10^{7}$ particules par seconde. Un gramme de radium en émettant 5 , 4.10 $0^{10}$, la quantité de polonium était équivalente à celle en équilibre avec 4,1 mgr. de Ra.

La quantité d'hélium produite par le polonium devait être proportionnelle à l'expression

$$
\frac{1}{\lambda}\left(1-e^{-\lambda t}\right)
$$

où $\lambda$ est la constante radioactive du polonium et $t$ le temps exprimé en jours. I.a valeur de cette expression, pour $t=128$, est 96, c'est-à-dire que la quantité formée en 128 jours est 96 fois plus grande que la quantité formée par jour au début. Le volume total d'hélium produit devait donc être

$$
0,0041 \times 0,107 \times 96=0,042 \mathrm{~mm}^{3} .
$$

Ainsi, la quantité trourée en réalité n’a été que le quart environ de la quantité théorique; ce désaccord est dû sans doute à ce qu'une très grande partie des particules $\alpha$ était restée occluse dans le cuivre. La production d'hélium par le polonium n'a pu par conséquent être établie que qualitativement.
Production d'hélium par le radium D. Quoique le Ral) n'émitte pas de particules $\alpha$, cependant, lorsqu'il est abandonni à Jui-même pendant assez longtemps, il donne naissance au RaD et au RaF (polonium), et l'on doit s'attendre à ce que ce dernier produise de l'hélium. Le sulfate de plomb séparé de la solution polonifère, de la manière décrite plus haut, et contenant le radioplomb ou RaI), f.ut maintenu enfermé dans un tube évacué de verre dur pendant 110 jours environ. Le sulfate fut alors chauffé et les gaz retirés; le gaz non condensable par le charbon fit voir un bon spectre d'hélium; toutefois son volume n'a pas été déterminé.

Rẻsumé des résultats. - La production d'hélium par le radium a été mesuréc avec la plus grande précision; elle s'est trouvée être de $0,107 \mathrm{~mm}^{3}$ environ par jour et par gramme de Ra (ćlément), soit $156 \mathrm{~mm}^{5}$ par an et par gr de Ra en équilibre avec ses premiers produits (émanation, RaA, RaC); cette quantité s'accorde aussi bien que possible avec la production de $158 \mathrm{~mm}^{3}$ par an, calculée par Rutherford et Geiger sur la base de leurs expériences relatives à la numération des particules $\alpha$ émises par le radium.

On a mesuré aussi la quantité d'hélium produite par la décomposition d'une quantité donnée d'émanations du radium; la production observée a correspondu complètement à celle qu'on pouvait prévoir théoriquement. On a observé en outre la production d'hélium par des préparations de polonium et de radioplomb.

[Traduit par L. Kolowrat.] 